\title{
Cognitive behavioral therapy for insomnia
}

\section{comorbid with COPD is feasible with preliminary evidence of positive sleep and fatigue effects}

This article was published in the following Dove Press journal:

International Journal of COPD

23 November 201I

Number of times this article has been viewed

\section{Mary C Kapella' \\ James J Herdegen² \\ Michael L Perlis ${ }^{3}$ \\ Joan L Shaver ${ }^{4}$ \\ Janet L Larson ${ }^{5}$ \\ Julie A Law ${ }^{2}$ \\ David W Carley'}

'Center for Narcolepsy, Sleep and Health Research, Department of

Biobehavioral Science, College of Nursing, ${ }^{2}$ Section of Pulmonary,

Critical Care, Sleep and Allergy,

College of Medicine, University of

Illinois at Chicago, Chicago, IL, USA;

${ }^{3}$ Department of Psychiatry, University of Pennsylvania, Philadelphia, PA, USA;

${ }^{4}$ College of Nursing, University of Arizona, Tucson, AZ, USA; ${ }^{5}$ Division of Acute, Critical and Long Term Care Programs, University of Michigan

School of Nursing, Ann Arbor, MI, USA
Background: Many people with COPD report difficulties falling asleep or staying asleep, insufficient sleep duration, or nonrestorative sleep. Cognitive behavioral therapy for insomnia (CBT-I) has proved effective not only in people with primary insomnia but also in people with insomnia comorbid with psychiatric and medical illness (eg, depression, cancer, and chronic pain). However, CBT-I has rarely been tested in those with COPD who have disease-related features that interfere with sleep and may lessen the effectiveness of such therapies. The purpose of this study was to determine the feasibility of applying a CBT-I intervention for people with COPD and to assess the impact of CBT-I on insomnia severity and sleep-related outcomes, fatigue, mood, and daytime functioning.

Methods: The study had two phases. In Phase 1, a 6-weekly session CBT-I intervention protocol in participants with COPD was assessed to examine feasibility and acceptability. Phase 2 was a small trial utilizing a prospective two-group pre- and post-test design with random assignment to the six-session CBT-I or a six-session wellness education (WE) program to determine the effects of each intervention, with both interventions being provided by a nurse behavioral sleep medicine specialist.

Results: Fourteen participants (five in Phase 1 and nine in Phase 2) completed six sessions of CBT-I and nine participants completed six sessions of WE. Participants indicated that both interventions were acceptable. Significant positive treatment-related effects of the CBT-I intervention were noted for insomnia severity $(P=0.000)$, global sleep quality $(P=0.002)$, wake after sleep onset $(P=0.03)$, sleep efficiency $(P=0.02)$, fatigue $(P=0.005)$, and beliefs and attitudes about sleep $(P=0.000)$. Significant positive effects were noted for depressed mood after WE $(P=0.005)$

Conclusion: Results suggest that using CBT-I in COPD is feasible and the outcomes compare favorably with those obtained in older adults with insomnia in the context of other chronic illnesses.

Keywords: CBT-I, emphysema, chronic bronchitis, sleep disturbance

\section{Introduction}

It is estimated that up to $50 \%$ of people with COPD report difficulty falling asleep, staying asleep, or unrefreshing sleep (ie, insomnia). ${ }^{1,2}$ This high prevalence rate of insomnia, nearly double that found in the population at large, may be due to several factors including nocturnal dyspnea, diminished physical activity, reduced time spent outside and/or reduced exposure to bright light, and/or the expansion of sleep opportunity as a means of coping with disease-related fatigue. The combination of these factors suggests that insomnia in the context of COPD may be responsive to cognitive behavioral therapy for insomnia (CBT-I). Evaluating the efficacy of this approach is
Correspondence: Mary C Kapella University of Illinois at Chicago, 845 South Damen Ave, Chicago, IL $606 / 2-7350$, USA

$\mathrm{Tel}+\mathrm{I} 3123553150$

$\mathrm{Fax}+\mathrm{I} 3129964979$

Email mkapelI@uic.edu 
additionally important in view of the natural tendency of clinicians to avoid using hypnotics in COPD patients (as they may cause respiratory depression and a decreased arousal response to hypercapnia). ${ }^{3}$

CBT-I is a nonpharmacological therapy, which is provided over several sessions and commonly includes stimulus control, sleep restriction, sleep hygiene, training in relaxation, and cognitive therapy (see Table 1). To date, CBT-I has been exhaustively evaluated in patients with primary insomnia. In this context, CBT-I was found to be as efficacious as pharmacotherapy during acute treatment and yielded more durable benefits following treatment. ${ }^{4-11}$ That is, the clinical gains of CBT-I are, unlike those of pharmacotherapy, sustained once treatment has ceased.

In recent years, a variety of studies have assessed the efficacy of CBT-I for insomnia comorbid with psychiatric and medical illness. ${ }^{12,13}$ These studies have shown CBT-I to be effective for the insomnia that co-occurs with a variety of illnesses including depression, ${ }^{14}$ osteoarthritis, ${ }^{15}$ cancer, ${ }^{16,17}$ fibromyalgia, ${ }^{18}$ chronic pain, ${ }^{19-21}$ cardiopulmonary disease, ${ }^{22}$ and sleep disordered breathing. ${ }^{23}$ Remarkably, the preto post-treatment effect sizes are, more often than not, comparable with those for primary insomnia and in some cases larger. Finally, there is growing evidence that improved sleep following CBT-I influenced the clinical course of the comorbid illnesses. The data in this regard are preliminary but nonetheless suggestive, as CBT-I has been shown to result in higher response and remission rates when used as an adjuvant treatment with antidepressant therapy ${ }^{24}$ and to increase pain tolerance in patients with chronic pain. ${ }^{20}$

Table I Description of the CBT-I protocol

\begin{tabular}{|c|c|}
\hline Session I & T provides CBT-I overview. P completes daily sleep diary \\
\hline Session 2 & $\begin{array}{l}\mathrm{T} \text { and } \mathrm{P} \text { discuss sleep diary/actigraphy data, stimulus } \\
\text { control, and sleep restriction behaviors with bedtime } \\
\text { and arise time chosen based on sleep diary data and } \\
\text { sleep restriction homework }\end{array}$ \\
\hline Session 3 & $\begin{array}{l}\mathrm{T} \text { and } \mathrm{P} \text { discuss sleep diary/actigraphy, any problems } \\
\text { with homework, adjustments in sleep prescription, and } \\
\text { sleep hygiene measures }\end{array}$ \\
\hline Session 4 & $\begin{array}{l}\text { T and P discuss sleep diary/actigraphy data, adjustments } \\
\text { to sleep prescription, sleep medications, and cognitive } \\
\text { techniques for managing everyday and sleep-loss worries }\end{array}$ \\
\hline Session 5 & $\begin{array}{l}\mathrm{T} \text { and } \mathrm{P} \text { discuss sleep diary/actigraphy data, adjustments to } \\
\text { sleep prescription, and how to engage in two relaxation } \\
\text { techniques (imagery, progressive muscle relaxation) }\end{array}$ \\
\hline Session 6 & $\begin{array}{l}\mathrm{T} \text { and } \mathrm{P} \text { review progress and discuss ongoing techniques } \\
\text { to prevent insomnia relapse }\end{array}$ \\
\hline
\end{tabular}

Abbreviations: CBT-I, cognitive behavioral therapy for insomnia; T, therapist; P, participant.
Several factors suggest a clear need to evaluate the effects of CBT-I in the context of COPD. Behavioral factors may, in part, be responsible for the occurrence of chronic insomnia with COPD. Many physicians are reluctant to prescribe hypnotics to patients with COPD and, in both primary (insomnia disorder) and secondary insomnia (comorbid insomnia), CBT-I has demonstrated efficacy. Therefore, the purposes of this study were to determine the feasibility of applying a CBT-I intervention for people with mild to severe COPD manifestations and to assess the effects of CBT-I on insomnia severity, sleep-related outcomes (self-reported and actigraphic sleep quality indicators, sleep beliefs/attitudes), fatigue, mood, and daytime functioning.

\section{Methods Design}

This preliminary study was conducted in two phases. In Phase 1, a 6-weekly session CBT-I intervention protocol was tested with five participants with COPD to examine feasibility and acceptability of the CBT-I intervention. An audiotaped interview was conducted with each participant at the end of the six-session intervention to explore perceptions about the length and components of the CBT-I intervention and participants' rating of acceptability to the intervention. Phase 2 was structured as a small trial, utilizing a prospective two-group pre- and post-test design with stratified random assignment of 18 participants to the six-session CBT-I or a sixsession wellness education (WE) program. Randomization was stratified by COPD disease severity and gender.

\section{Setting and sample}

The research was approved by the local institutional review board and all participants provided written consent to participate. Participants with mild to severe $\mathrm{COPD}^{25}$ and no other major health problems were recruited from the Chicago area through print advertisements and word of mouth. The inclusion criteria were: abnormal $\mathrm{FEV}_{1} / \mathrm{FVC}$ ratio $<70 \%$, age $\geq 45$ years, stable clinical condition (no major exacerbation of COPD within the previous month) and self-reported difficulty initiating or maintaining sleep, waking up too early or poor quality sleep. Participants were excluded if they showed evidence of restrictive lung disease, history of asthma, a major sleep disorder other than insomnia (sleep apnea or periodic limb movements), hypnotic medication use, resting hypoxemia, the presence of a potentially debilitating disease such as cancer, congestive heart failure, kidney disease, liver failure or cirrhosis, a self-reported current diagnosis of major depression, or currently participating in pulmonary rehabilitation. 


\section{Screening and sample}

The initial screening was by telephone followed by a first screening visit to the authors' pulmonary research lab and a second screening visit for an overnight polysomnography. During the first screening visit, participants were assessed for COPD disease severity using pulmonary function testing. Two instruments were administered: the Sleep Impairment Inde ${ }^{26}$ to assess for inclusion and the Hospital Anxiety and Depression Scale (HADS ${ }^{27}$ criteria to assess for exclusion. At the second screening visit participants were screened for significant sleep apnea and periodic limb movements during sleep.

\section{Instruments}

\section{Assessment}

Participants were assessed for COPD disease severity using pulmonary function testing, which was performed on the VMAX Encore 22 (Viasys Healthcare, Inc, Yorba Linda, CA) according to established standards. ${ }^{28}$

The Sleep Impairment Index (SII), ${ }^{26,29}$ also known as the Insomnia Severity Index, was administered to select participants with at least a moderate perception of insomnia. The index includes seven items, each rated on a five-point scale ( $0=$ not at all, $4=$ extremely) to evaluate: (1) sleep onset severity, (2) sleep maintenance, (3) early morning awakening problems, (4) satisfaction with current sleep pattern, (5) interference with daily functioning, (6) impairment attributed to the sleep problem, and (7) level of distress caused by the sleep problem. Participants scoring $<10$ were excluded from the study. ${ }^{29}$

HADS, ${ }^{27,30}$ with a cutoff score of eleven for the depression scale, was used to screen for depressive symptoms; this scale includes 14 items rated on a four-point Likert scale.

The Epworth Sleepiness Scale was used to measure the severity of daytime sleepiness. Respondents rated eight items regarding the likelihood of dozing in sedentary situations on a scale from 0 (never) to 3 (high chance).

Participants were screened for major sleep disorder using a one-night polysomnography (PSG) with pulse oximetry to rule out sleep-related breathing and movement disorders associated with arousals. A standard PSG montage, including electroencephalographic, electromyographic, electrooculographic, electrocardiographic, respiratory, and limb movement monitoring, was used. Sleep stages were scored by an experienced technician according to standardized criteria. ${ }^{31}$ People with an apnea/hypopnea index of $>10$, and those with $>10$ periodic limb movements associated with arousal per hour of sleep were excluded. The PSG montage included pulse oximetry - used to exclude participants with hemoglobin saturation $\leq 85 \%$ for more than 5 minutes during sleep.

\section{Outcome}

The CBT-I intervention was assessed for feasibility using an interview and questionnaire. Participants were asked about their reactions to the length and components of the CBT-I intervention in an audiotaped interview. The eight-item Insomnia Treatment Acceptability Scale (ITAS), ${ }^{26}$ modified, was used to examine treatment acceptability. Respondents scored each item from 0 (not at all acceptable) to 4 (very acceptable) to rate if the rationale made sense, how acceptable the treatment was for them, suitability for their sleep problem, and expected effectiveness for their sleep problem. For the study sample, Cronbach's alpha for the ITAS was 0.85 .

The Sleep Impairment Index ${ }^{26,29}$ was used to measure insomnia severity. Total scores range from 0 to 28 , with high scores indicating greater insomnia severity. For the study sample, the Cronbach's alpha coefficient was 0.86 .

Perceived sleep quality was measured using the Pittsburgh Sleep Quality Index (PSQI) and a sleep diary. ${ }^{26} \mathrm{PSQI}^{32,33}$ is a 24-item instrument that measures perceived sleep quality with a global sleep quality index (the sum of seven component scores). Higher scores indicate poorer sleep quality. For the study sample, the PSQI Cronbach's alpha was 0.76.

Participants also completed a daily sleep diary ${ }^{26}$ throughout the study from which several sleep quality indicators were derived. These included sleep latency, minutes to fall asleep, frequency and duration of awakenings after sleep onset (WASO), time in bed trying to sleep, and total sleep time (TST). Sleep efficiency (SE) was computed as the ratio of total sleep time to time spent in bed and multiplied by 100 . Sleep variables were scored for each night and a weekly mean was computed. ${ }^{34,35}$

Actigraphic sleep quality indicators were assessed using the AW-2 Actiwatch (Minimitter, Philips Respironics, Andover, MA). It was programmed for start time and data collection interval and data were retrieved for analysis via a personal computer interface using scoring software provided with the accelerometer. The accelerometer was worn at night on the nondominant wrist for a minimum of 3 days at baseline and again at the end of the intervention and the report was reviewed at each session. Scores for the same variables as from the sleep diary were derived. ${ }^{36}$

Sleep-related beliefs and attitudes were measured using the Dysfunctional Beliefs and Attitudes about Sleep Scale. The scale consists of 30 items that measure sleep-related beliefs or attitudes in five themes: (1) misattributions 
or amplification of the consequences of insomnia, (2) diminished perception of control and predictability of sleep, (3) unrealistic sleep expectations, (4) misconceptions about the causes of insomnia, and (5) faulty beliefs about sleeppromoting practices. It includes a 0-10 Likert scale ranging from "strongly disagree" to "strongly agree." Higher scores indicate more dysfunctional beliefs. ${ }^{37,38}$ For the study sample, Cronbach's alpha coefficient was 0.86 .

Fatigue was measured using two instruments. The fouritem Chronic Respiratory Disease Questionnaire Fatigue Scale (CRQ-F), ${ }^{39-41}$ was used to measure the frequency and intensity of fatigue. The total score was derived from a mean of the four items, each scored on a seven-point scale. Lower scores indicated more fatigue. A change of at least 0.5 per item was considered the minimum for clinical significance and a change of one per item was considered of moderate clinical significance. ${ }^{42}$ For the study sample, Chronbach's alpha coefficient was 0.91 . The seven-item fatigue-inertia subscale of the Profile of Mood States (POMS-F) was also used to measure fatigue. ${ }^{43,44}$ For the study sample, the Cronbach's alpha coefficient for the POMS-F was 0.89.

Anxious and depressed moods were assessed using the tension-anxiety (POMS-A) and depression-dejection (POMS-D) subscales of the POMS. ${ }^{43}$ For the study sample, Cronbach's alpha coefficients for the POMS-A and the POMS-D were 0.86 and 0.92 , respectively.

Perceived daytime function was measured by the Functional Performance Inventory (FPI). ${ }^{45,46}$ The FPI is a 65 -item instrument that measures performance of day-to-day activities. Six subscales include body care, maintaining the household, physical exercise, recreation, spiritual activities, and social interaction. The FPI potential total score range is $0-3$, with higher scores reflecting better daytime functioning. The Cronbach's alpha for the FPI was 0.97 in this study.

\section{Procedures (intervention and control condition)}

The CBT-I intervention protocol followed Perlis and colleagues' insomnia treatment protocol, with modifications to the sleep restriction and cognitive therapy components (described below). ${ }^{47}$ Sessions were provided by a nurse behavioral sleep medicine specialist. Behavioral sleep medicine training included coursework in cognitive behavioral intervention, attending a 2.5-day seminar on insomnia and CBT-I, observing cases where CBT-I was delivered by a licensed clinical psychologist, and delivering CBT-I under supervision of a physician board certified in sleep medicine. Consultations with a licensed clinical psychologist experienced in
CBT-I were obtained as needed during the training period. Intervention sessions were audiotaped and sent to a licensed clinical psychologist experienced in CBT-I who assessed the sessions for fidelity.

Early on, it was recognized that CBT-I participants benefited from seeing the actigraphy report during their session and found it worthwhile to wear the device at night during the intervention period. During each session, the actigraphy and the sleep diary data were reviewed and time to bed and out of bed were discussed to score the actigraphy, provide feedback, and encourage participants to adhere to "homework" and completing the sleep diary. Thus, each session included a review of the sleep diary and actigraphy data, a didactic/ interactive presentation and discussion to answer questions, and work on problems encountered while implementing the study homework in the previous week.

In session 1, the study components were described and the Spielman model of insomnia was discussed..$^{48}$ The main components of the intervention, stimulus control and sleep restriction, were introduced during session 2. Information on ways to optimize sleep was given. For instance, participants were advised to get out of bed if awake longer than 15 minutes and go into another room (to reinforce the relationship between bed and sleep), returning to bed when they again felt sleepy. Discussion also centered around bedtimes and rising times and participants were recommended to limit their time in bed to the amount of time they were sleeping, according to their sleep diary, to consolidate their sleep, but not less than 5 hours per night. The prescribed "time to bed" for the next week was set at each session by setting time in bed to an amount approximately equal to average sleep ability (average TST as assessed by the sleep diary), where "lights off" was determined by subtracting the average TST from the desired wakeup (time out of bed) time. In general, the recommended bedtime was moved earlier weekly by $15-30$ minutes, the amount of time determined by agreement of the therapist and participant, if there was an improvement in sleep efficiency, defined as $\geq 85 \%$ sleep efficiency. Bedtime was kept the same if sleep efficiency was between $80 \%-84 \%$ and delayed if $\leq 79 \%$. This differs from the sleep restriction procedure described in Perlis et al in which the time is moved earlier by 15 minutes without variation. ${ }^{47}$

Adherence to stimulus control and sleep restriction was assessed at each session using the sleep diary and/or the actigraph. Evaluation of adherence to stimulus control was assessed by examining responses to a sleep diary question, which asked how the participant managed periods awake during the night. Evaluation of adherence to sleep restriction 
was assessed by comparing the recommended time in bed with the reported time in bed.

The remainder of each session included a didactic/ interactive presentation of a new topic followed by discussion. Topics included sleep hygiene, including information about the effect of temperature and light (session 3); information about medications for sleep (session 4); a cognitive component focusing on worry and stress, including ways to work through worries ${ }^{49}$ (note: this component differs from the procedure described in Perlis et $\mathrm{al}^{47}$ ). Because people with COPD struggle with anxiety and worries, ${ }^{50}$ in addition to cognitive therapy on reducing catastrophic thoughts about sleep, work also focused on strategies to manage everyday worries and relaxation techniques (session 5), and a review and discussion of relapse prevention (session 6).

WE sessions were focused on topics related to COPD. Each session was conducted by the same nurse behavioral sleep medicine specialist who provided the CBT-I program. Topics included how the lungs work, medications commonly prescribed for people with COPD, inhalers and other breathing equipment, breathing techniques, managing a cold or flu and vaccination information, and environmental influences.

\section{Analysis}

The feasibility interview data were transcribed from the audiotapes, and the qualitative data were analyzed using inductive methods for content analysis. Quantitative data were analyzed with descriptive statistics and paired $t$-tests using PASW Statistics 18 (v 18.0.0; SPSS, Inc, Chicago, IL). Item missing values were replaced using mean substitution. For unit missing data, it was determined whether missing data were missing completely at random. Missing data occurred randomly except in one subject in the CBT-I group where complete sleep diary data were missing. Bivariate relationships were examined with Pearson's correlations. Analysis of variance (ANOVA) was used to compare baseline characteristics for the groups in terms of age, gender, and pulmonary function tests. Difference scores between pre- and postintervention were used to calculate Cohen's $d$ within effect size as: mean score difference pre- to post-divided by the pooled standard deviation (SD). Phase 1 and 2 CBT-I data were combined for selected analyses as described in the Results section.

\section{Results}

A total of 90 potential participants completed the initial telephone screening and 50 people were excluded at the first laboratory screening visit. Twenty-four people were found to be eligible, 14 completed six sessions of CBT-I and nine completed six sessions of WE. One participant was lost before baseline as they were unable to be contacted. Figure 1 shows a flow diagram of the study enrollment.

Table 2 describes baseline characteristics for participants who completed the study. No significant differences were noted between groups in the baseline characteristics, including for nighttime apnea/hypopnea index, periodic limb movement arousal index, $\mathrm{SaO}_{2}$, or for excessive daytime sleepiness. Participants had mild to moderate COPD according to the Global initiative for chronic Obstructive Lung Disease (GOLD) standard; ${ }^{51}$ GOLD stage I $(n=4)$, GOLD stage II $(n=12)$, and GOLD stage III $(n=7)$.

\section{CBT-I feasibility}

Acceptable feasibility was judged by intervention participation and self-measurement rates; reports on the ITAS and for the subset who were interviewed on audiotape.

Participants in Phase $1(n=5)$ who completed the audiotaped interview at the end of the study verbalized that the CBT-I program helped them achieve more and better sleep. In general they agreed with the average 1-hour length of the sessions but 2/5 participants indicated that they would have liked more sessions; one indicated that six sessions was about right and one person suggested that the program be offered online. Participants indicated that their family members were supportive of their CBT-I activities. Selected comments from participants in the CBT-I:

- "I got sleep. I got sleep. I slept. I was able to sleep."

- "I'm kind of surprised that it helped but it did."

- "I think just learning that it's actually part of things that I can control rather than saying that it is just you know, part of nature, part of the aging process. There were actually things that I could control to help me get a better night's sleep."

All participants in Phase 2 were willing to be randomized to either intervention. There were no dropouts from the study after baseline occurred and no sessions were missed, although some were rescheduled due to holidays, weather, and personal reasons. One participant experienced symptoms of an acute exacerbation of COPD and was treated by his physician between CBT-I sessions. This participant took part in the weekly CBT-I sessions without interruption. Twenty-two people completed the sleep diary on paper, mostly due to lack of computer access or computer literacy, and one person completed the online version of the identical sleep diary. One participant refused to complete the sleep diary during the 


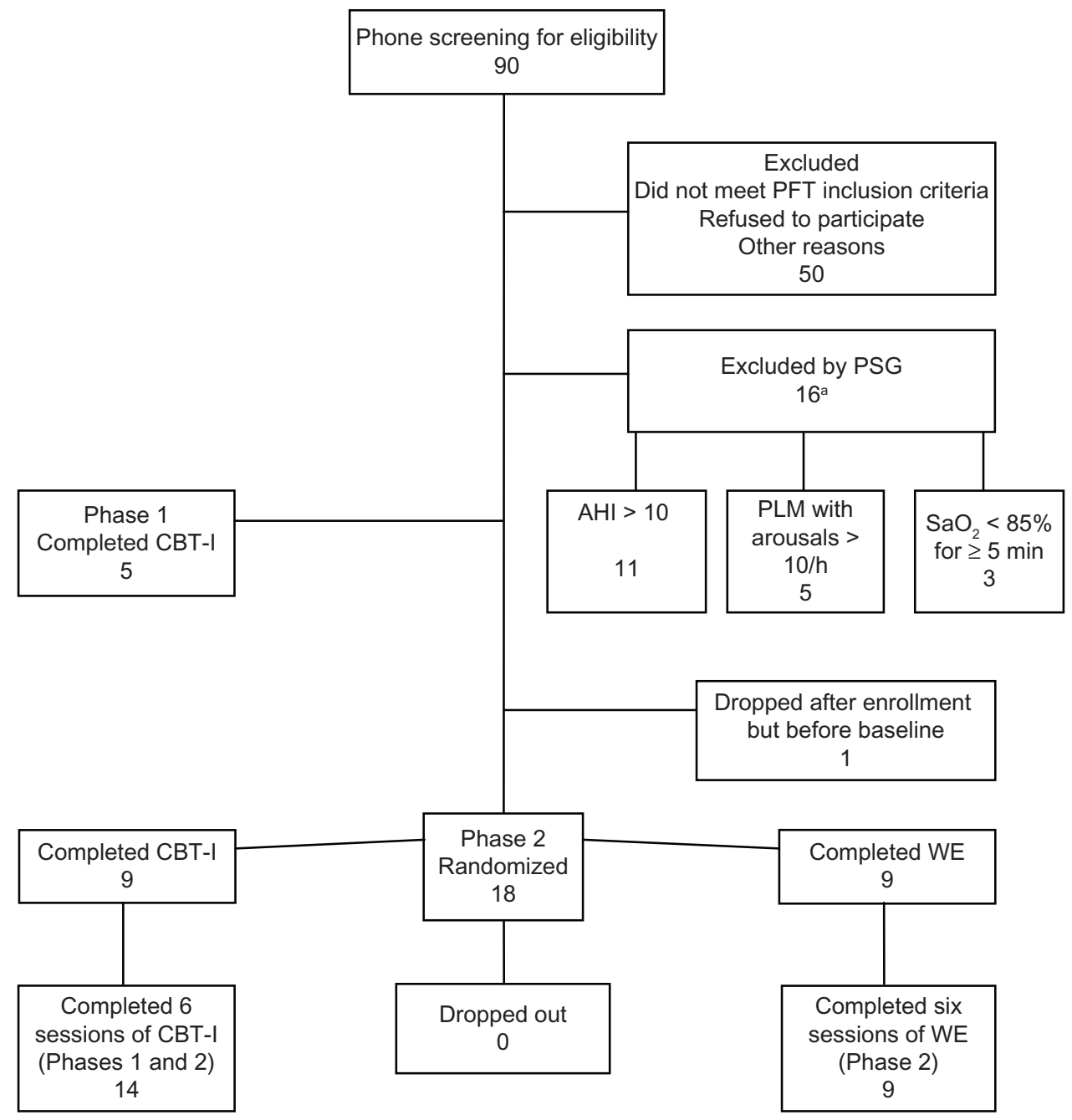

Figure I Flow diagram of the study enrollment.

Note: ${ }^{a}$ Three participants were excluded by more than one criterion.

Abbreviations: AHI, apnea/hypopnea index; CBT-I, cognitive behavioral therapy for insomnia; PFT, pulmonary function testing; PLM, periodic limb movement; PSG, programmable sound generator; WE, wellness education.

intervention period but wore the actigraph at night throughout the study and participated in all sessions.

All participants in Phases 1 and $2(n=14)$ reported that the CBT-I intervention was acceptable on the ITAS (range $0-4$; mean $[\mathrm{M}]=3.1, \mathrm{SD}=0.6$ ). The WE group also reported that their intervention was acceptable but acceptance was somewhat lower and more variable $(\mathrm{M}=2.7, \mathrm{SD}=0.8$; see Figure 2). Subjects in the WE group had lower mean scores (range 0-4) compared with the CBT-I group on the item measuring expectation for how effective the treatment would be in the short term $(\mathrm{M}=2.2, \mathrm{SD}=1$, vs $\mathrm{M}=3.1, \mathrm{SD}=0.7)$.

\section{CBT-I outcomes}

Descriptive statistics and pre- and postintervention outcomes for the sleep-related measures are presented in Table 3 . Participants reported moderate levels of insomnia at baseline.
In general when compared with the CBT-I group, participants in the WE group reported better sleep preintervention but had worse preintervention sleep on actigraphy. Correlations between sleep diary and actigraphy variables at baseline were moderate to low for sleep latency, wake after sleep onset, total sleep time, and sleep efficiency $(r=0.66,0.18,0.28$, and 0.13 respectively).

For the CBT-I group ( $\mathrm{n}=14)$, significant within-group differences were noted for insomnia severity $(P=0.000)$, PSQI global sleep quality $(P=0.002)$, sleep diary WASO $(P=0.030)$, sleep diary SE $(P=0.017)$, actiwatch SE $(P=0.028)$, and dysfunctional beliefs and attitudes about sleep $(P=0.000)$. No significant within-group differences in subjectively or objectively measured sleep indicators were seen pre- or post-WE. All other sleep diary and actigraphic variables showed mean changes in an improved direction 
Table 2 Sample characteristics at baseline

\begin{tabular}{|c|c|c|c|}
\hline Characteristics & $\begin{array}{l}\text { All subjects }(n=23)^{a} \\
\text { Mean } \pm \text { SD }\end{array}$ & $\begin{array}{l}\text { CBT-I }(n=9)^{b} \\
\text { Mean } \pm \text { SD }\end{array}$ & $\begin{array}{l}\text { Wellness education }(n=9)^{b} \\
\text { Mean } \pm \text { SD }\end{array}$ \\
\hline Age (years) & $63 \pm 10$ & $65 \pm 9$ & $60 \pm 10$ \\
\hline Men/women (n) & $19 / 4$ & $7 / 2$ & $7 / 2$ \\
\hline $\mathrm{FEV}_{1}$, \% predicted & $62 \pm 18$ & $62 \pm 21$ & $59 \pm 18$ \\
\hline $\mathrm{FEV}_{1} / \mathrm{FVC}$ & $50 \pm 10$ & $47 \pm 7$ & $53 \pm 14$ \\
\hline BMI & $25 \pm 5$ & $27 \pm 6$ & $24 \pm 3$ \\
\hline \multicolumn{4}{|l|}{ Race (n) } \\
\hline African-American & 5 & I & 4 \\
\hline Caucasian & 18 & 8 & 5 \\
\hline Other & 0 & 0 & 0 \\
\hline \multicolumn{4}{|l|}{ Educationc } \\
\hline Less than high school & 2 & I & 0 \\
\hline High school & 5 & 4 & 1 \\
\hline Some college & 8 & 4 & 4 \\
\hline College & 7 & I & 4 \\
\hline \multicolumn{4}{|l|}{ Employment $^{\mathrm{c}}$} \\
\hline Retired & 6 & 3 & 1 \\
\hline Part-time & 5 & 2 & 2 \\
\hline Full time & I & I & 0 \\
\hline Unemployed & 10 & 3 & 6 \\
\hline Smoking, pack-years & $46 \pm 20$ & $48 \pm 32$ & $4 I \pm 8$ \\
\hline PSG, apnea/hypopnea index & $4 \pm 3$ & $5 \pm 3$ & $3.5 \pm 2$ \\
\hline PSG, periodic limb movement arousal index (per hour) & $1.0 \pm 2$ & $0.47 \pm 0.6$ & $1.5 \pm 2$ \\
\hline PSG, mean $\mathrm{SaO}_{2}$ & $94 \pm 2$ & $95 \pm 3$ & $94 \pm 1$ \\
\hline PSG, minimum $\mathrm{SaO}_{2}$ & $86 \pm 5$ & $85 \pm 5$ & $86 \pm 5$ \\
\hline Excessive daytime sleepiness (Epworth Sleepiness Scale) & $9.2 \pm 5$ & $8.0 \pm 4.3$ & $\mathrm{II} \pm 5$ \\
\hline
\end{tabular}

Notes: ancludes all subjects (CBT-I and WE, Phases I and 2); bincludes subjects randomized to CBT-I or WE (Phase 2); 'data missing for one subject.

Abbreviations: BMI, body mass index; CBT-I, cognitive behavioral therapy for insomnia; FEV,/FVC, forced expiratory volume in I second/forced vital capacity; PSG, programmable sound generator; WE, wellness education; SD, standard deviation.

for the CBT-I group (Table 3). For the WE group, although not statistically significant, perceived and actigraphic WASO, TST, and SE also moved in an improved direction. Dysfunctional Beliefs and Attitudes about Sleep Scale scores at the end of the study $(n=23)$ were significantly associated

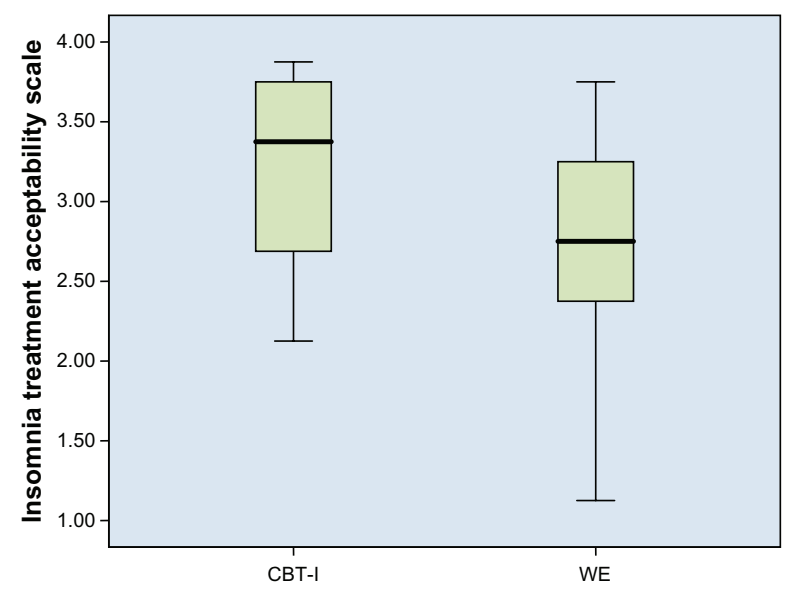

Figure 2 Acceptability of treatments (CBT-I and WE).

Abbreviations: CBT-I, cognitive-behavioral therapy for insomnia; WE, wellness education. with insomnia severity $(\mathrm{r}=0.61, P=0.002)$, PSQI global sleep quality ( $\mathrm{r}=0.60, P=0.002)$, CRQ-F ( $\mathrm{r}=-0.60$, $P=0.004)$, and POMS-F $(\mathrm{r}=0.60, P=0.003)$.

Descriptive statistics and pre- and postintervention outcomes for the fatigue, mood, and daytime functioning measures are presented in Table 4. Participants reported moderate levels of fatigue at baseline. Significant improvement in CRQ-F scores after CBT-I $(P=0.005)$ was noted and this was corroborated by a trend in the POMS-F. Of CBT-I and WE participants, $57 \%$ and $46 \%$, respectively, had at least a 1.00 change in CRQ-F after the intervention, suggesting clinical significance. For the CBT-I group, neither the POMS-A nor POMS-D subscales showed statistically significant improvement from pre- to postintervention. However, for the WE group, significantly improved scores on the POMS-D subscale were seen $(P=0.005)$. No significant improvements in either group were noted from pre- to postintervention for daytime functioning.

\section{Discussion}

Results of this study support the feasibility and acceptability of the CBT-I intervention for people with COPD and insomnia. 
Table 3 Descriptive statistics and effect size for sleep outcomes: all CBT-la (Phases I and 2, $n=14$ ), CBT-I $($ Phase 2, $n=9$ ), WE ${ }^{\mathrm{b}}$ (Phase 2, $\mathrm{n}=9$ )

\begin{tabular}{|c|c|c|c|c|c|}
\hline $\begin{array}{l}\text { Variable } \\
\text { Measure }\end{array}$ & $\begin{array}{l}\text { Preintervention } \\
\text { Mean } \pm \text { SD }\end{array}$ & $\begin{array}{l}\text { Postintervention } \\
\text { Mean } \pm \text { SD }\end{array}$ & $\begin{array}{l}\text { Within-group difference } \\
\text { Mean } \pm \text { SD }\end{array}$ & $P$ value & $\begin{array}{l}\text { Within-group } \\
\text { effect size }\end{array}$ \\
\hline \multicolumn{6}{|c|}{ Subjective sleep outcomes } \\
\hline \multicolumn{6}{|c|}{ Insomnia severity (SII) $)^{26,29}$} \\
\hline All CBT-I & $16.6 \pm 4.9$ & $10.6 \pm 4.1$ & $-5.9 \pm 3.3$ & 0.000 & I.79* \\
\hline CBT-I & $15.9 \pm 5.6$ & $10.7 \pm 4.4$ & $-5.2 \pm 3.7$ & 0.003 & $1.40 *$ \\
\hline WE & $13.9 \pm 4.5$ & $12.4 \pm 3.3$ & $-1.4 \pm 3.7$ & 0.273 & 0.38 \\
\hline \multicolumn{6}{|c|}{ Global sleep quality (PSQI) ${ }^{32,33}$ (lower is better) } \\
\hline All CBT-I & $11.0 \pm 3.6$ & $6.5 \pm 3.4$ & $-4.5 \pm 4.4$ & 0.002 & $1.02 *$ \\
\hline CBT-I & $10.1 \pm 3.7$ & $7.2 \pm 3.7$ & $-2.9 \pm 4.2$ & 0.068 & 0.69 \\
\hline WE & $7.1 \pm 2.4$ & $7.5 \pm 2.4$ & $0.44 \pm 1.9$ & 0.512 & 0.23 \\
\hline \multicolumn{6}{|c|}{ Dysfunctional beliefs and attitudes about sleep (DBAS 30) } \\
\hline All CBT-I & $4.1 \pm 1.5$ & $3.1 \pm 1.7$ & $-1.0 \pm .74$ & 0.000 & $1.35^{*}$ \\
\hline CBT-I & $4.1 \pm 1.8$ & $3.2 \pm 2.0$ & $-0.85 \pm 0.80$ & 0.013 & $1.06^{*}$ \\
\hline WE & $3.7 \pm 0.94$ & $3.4 \pm 1.4$ & $-0.32 \pm 0.91$ & 0.317 & 0.35 \\
\hline \multicolumn{6}{|c|}{ Sleep continuity, sleep diary ${ }^{26}$} \\
\hline \multicolumn{6}{|c|}{ Sleep latency $(\mathrm{min})$} \\
\hline All CBT-I & $32 \pm 50$ & $17 \pm 16$ & $-15 \pm 34$ & 0.136 & 0.44 \\
\hline CBT-I & $37 \pm 60$ & $19 \pm 19$ & $-|8 \pm 4|$ & 0.219 & 0.44 \\
\hline WE & $22 \pm 18$ & $23 \pm 11$ & $1 \pm 9$ & 0.734 & 0.11 \\
\hline \multicolumn{6}{|c|}{ Wake after sleep onset (min) } \\
\hline All CBT-I & $70 \pm 56$ & $30 \pm 17$ & $-39 \pm 57$ & 0.030 & $0.68^{*}$ \\
\hline CBT-I & $68 \pm 38$ & $34 \pm 19$ & $-34 \pm 4 I$ & 0.037 & $0.83^{*}$ \\
\hline WE & $59 \pm 63$ & $38 \pm 23$ & $-21 \pm 53$ & 0.272 & 0.40 \\
\hline \multicolumn{6}{|c|}{ Total sleep time (min) } \\
\hline All CBT-I & $365 \pm 82$ & $404 \pm 55$ & $40 \pm 76$ & 0.080 & 0.53 \\
\hline CBT-I & $361 \pm 68$ & $408 \pm 57$ & $47 \pm 67$ & 0.071 & 0.70 \\
\hline WE & $382 \pm 114$ & $448 \pm 114$ & $66 \pm 106$ & 0.099 & 0.62 \\
\hline \multicolumn{6}{|c|}{ Number of awakenings } \\
\hline All CBT-I & $2.2 \pm 1$ & $1.9 \pm 0.91$ & $-0.35 \pm 0.90$ & 0.184 & 0.39 \\
\hline CBT-I & $2.6 \pm 1$ & $2.1 \pm 0.88$ & $-0.44 \pm 0.99$ & 0.223 & 0.44 \\
\hline WE & $2.1 \pm 1$ & $2.0 \pm 1.4$ & $-0.09 \pm 1.0$ & 0.248 & 0.09 \\
\hline \multicolumn{6}{|c|}{ Sleep efficiency (\%) } \\
\hline All CBT-I & $78 \pm 17$ & $89 \pm 6$ & $11 \pm 14$ & 0.017 & $0.78^{*}$ \\
\hline CBT-I & $78 \pm 15$ & $88 \pm 7$ & $11 \pm 12$ & 0.031 & $0.92 *$ \\
\hline WE & $82 \pm 13$ & $87 \pm 5$ & $4 \pm 13$ & 0.177 & 0.31 \\
\hline \multicolumn{6}{|c|}{ Sleep continuity, actigraphy } \\
\hline \multicolumn{6}{|c|}{ Wake after sleep onset (min) } \\
\hline All CBT-I & $98 \pm 60$ & $64 \pm 32$ & $-33 \pm 64$ & 0.070 & 0.52 \\
\hline CBT-I & $103 \pm 59$ & $68 \pm 39$ & $-35 \pm 71$ & 0.180 & 0.49 \\
\hline WE & $117 \pm 72$ & $105 \pm 56$ & $-13 \pm 65$ & 0.578 & 0.20 \\
\hline \multicolumn{6}{|c|}{ Total sleep time (min) } \\
\hline All CBT-I & $365 \pm 82$ & $405 \pm 55$ & $40 \pm 76$ & 0.080 & 0.53 \\
\hline CBT-I & $361 \pm 68$ & $408 \pm 57$ & $47 \pm 67$ & 0.071 & 0.70 \\
\hline WE & $268 \pm 90$ & $292 \pm 65$ & $25 \pm 51$ & 0.183 & 0.49 \\
\hline \multicolumn{6}{|c|}{ Number of awakenings } \\
\hline All CBT-I & $40 \pm 13$ & $36 \pm 12$ & $-4.3 \pm 15$ & 0.292 & 0.29 \\
\hline CBT-I & $45 \pm 15$ & $35 \pm 13$ & $-9.0 \pm 14$ & 0.070 & 0.64 \\
\hline WE & $33 \pm 10$ & $33 \pm 9$ & $0.35 \pm 8$ & 0.908 & 0.04 \\
\hline \multicolumn{6}{|c|}{ Sleep efficiency (\%) } \\
\hline All CBT-I & $72 \pm 10$ & $79 \pm 9$ & $7 \pm 10$ & 0.028 & $0.70 *$ \\
\hline CBT-I & $71 \pm 11$ & $78 \pm 11$ & $7 \pm 12$ & 0.120 & 0.58 \\
\hline WE & $61 \pm 17$ & $64 \pm 15$ & $3 \pm 13$ & 0.427 & 0.23 \\
\hline
\end{tabular}

Notes: ${ }^{a}$ Five CBT-I participants in Phase I and 9 participants in Phase 2; brandomized to CBT-I or WE; *P $<0.05$.

Abbreviations: CBT-I, cognitive behavioral therapy for insomnia; WE, wellness education; DBS 30, Dysfunctional Beliefs and Attitudes about Sleep Scale; SII, Sleep Impairment Index; PSQI, Pittsburgh Sleep Quality Index; SD, standard deviation. 
Table 4 Descriptive statistics and effect size for fatigue, mood, and daytime function

\begin{tabular}{|c|c|c|c|c|c|}
\hline $\begin{array}{l}\text { Variable } \\
\text { measure }\end{array}$ & $\begin{array}{l}\text { Preintervention } \\
\text { Mean } \pm \text { SD }\end{array}$ & $\begin{array}{l}\text { Postintervention } \\
\text { Mean } \pm \text { SD }\end{array}$ & $\begin{array}{l}\text { Within-group difference } \\
\text { Mean } \pm \text { SD }\end{array}$ & $P$ value & $\begin{array}{l}\text { Within-group } \\
\text { effect size }\end{array}$ \\
\hline \multicolumn{6}{|l|}{ Fatigue } \\
\hline \multicolumn{6}{|c|}{ CRQ-F $\mathrm{F}^{39-42}$ (lower indicates greater fatigue) } \\
\hline All CBT-I & $3.9 \pm 1.1$ & $4.6 \pm 0.95$ & $-0.75 \pm 0.84$ & 0.005 & $0.89 *$ \\
\hline CBT-I & $3.7 \pm 1.3$ & $4.5 \pm 1.0$ & $-0.75 \pm 0.87$ & 0.033 & $0.86 *$ \\
\hline$W^{b}$ & $3.5 \pm 1.2$ & $4.1 \pm 1.2$ & $-0.54 \pm 1.3$ & 0.313 & 0.41 \\
\hline \multicolumn{6}{|l|}{ POMS-F $\mathrm{F}^{43,44}$} \\
\hline All CBT-I & $8.6 \pm 5.7$ & $6.6 \pm 5.5$ & $-2.0 \pm 4.5$ & 0.122 & 0.44 \\
\hline CBT-I & $10.3 \pm 6.3$ & $7.7 \pm 5.6$ & $-2.7 \pm 4.5$ & 0.115 & 0.60 \\
\hline WE & $10.3 \pm 6.3$ & $10.0 \pm 8.1$ & $-0.33 \pm 3.3$ & $0.77 I$ & 0.10 \\
\hline \multicolumn{6}{|l|}{ Mood } \\
\hline \multicolumn{6}{|l|}{ POMS-A ${ }^{43,44}$} \\
\hline All CBT-I & $7.9 \pm 6.9$ & $6.6 \pm 4.6$ & $-1.4 \pm 4.7$ & 0.296 & 0.30 \\
\hline CBT-I & $9.4 \pm 8.2$ & $7.7 \pm 5.2$ & $-1.6 \pm 5.3$ & 0.376 & 0.30 \\
\hline WE & $8.6 \pm 3.7$ & $6.1 \pm 3.4$ & $-2.4 \pm 3.8$ & 0.091 & 0.63 \\
\hline \multicolumn{6}{|l|}{ POMS-D ${ }^{43,44}$} \\
\hline All CBT-I & $7.6 \pm 8.9$ & $4.8 \pm 7.1$ & $-2.8 \pm 5.3$ & 0.072 & 0.53 \\
\hline CBT-I & $9.9 \pm 10.3$ & $6.6 \pm 8.4$ & $-3.3 \pm 6.0$ & 0.133 & 0.55 \\
\hline WE & $10.4 \pm 8.2$ & $5.8 \pm 6.8$ & $-4.6 \pm 3.5$ & 0.005 & $\mathrm{I} .3 \mathrm{I}^{*}$ \\
\hline \multicolumn{6}{|c|}{ Daytime functioning } \\
\hline \multicolumn{6}{|c|}{$\mathrm{FPI}^{45,46}$ total score } \\
\hline All CBT-I & $2.2 \pm 0.40$ & $2.3 \pm 0.29$ & $0.09 \pm 0.24$ & 0.218 & 0.37 \\
\hline CBT-I & $2.3 \pm 0.33$ & $2.3 \pm 0.27$ & $0.01 \pm 0.15$ & 0.778 & 0.10 \\
\hline WE & $2.2 \pm 0.44$ & $2.2 \pm 0.47$ & $-0.03 \pm 0.16$ & 0.607 & 0.19 \\
\hline
\end{tabular}

Notes: All CBT-I $(n=14)$, CBT-I $(n=9)$, WE ${ }^{a}(n=9)$. ${ }^{a}$ Randomized to CBT-I or WE; ${ }^{b}=7$ due to missing data; ${ }^{*} P<0.05$.

Abbreviations: CBT-I, cognitive behavioral therapy for insomnia; WE, wellness education; CRQ-F, Chronic Respiratory Disease Questionnaire Fatigue Scale; POMS, Profile of Mood States; POMS-F, Profile of Mood States fatigue-inertia subscale; POMS-A, Profile of Mood States tension-anxiety subscale; POMS-D, Profile of Mood States depression-dejection subscale; FPI, Functional Performance Inventory; SD, standard deviation.

The data show that the recruiting and retaining of participants was successful, with high attendance rates. The study did, however, demonstrate a high percentage of primary sleep disorders (sleep apnea, periodic limb movements) that limited recruitment. It remains to be determined whether the $40 \%$ of subjects excluded for primary sleep disorders would benefit from CBT-I after successful treatment of their other sleep disorder.

In testing the effects of the CBT-I on sleep-related outcomes, the present findings are supportive of CBT-I being an effective intervention for people with COPD for improving perceived sleep quality and objective sleep indicators. Untreated, study participants with COPD reported moderate levels of insomnia ${ }^{29}$ and lower sleep quality at baseline compared with data in the literature about healthy older adults. ${ }^{52}$ This is indicative of their need for treatment to improve sleep quality. The effect trends for indicators of improved sleep are similar to those found in previous studies of CBT-I in older adults with comorbid insomnia. ${ }^{15,16,21,22,52}$ It was evident that the comparison intervention of WE had positive effects but many of the within-group effect sizes associated with the CBT-I intervention were at least twice as large as those in the WE group.
When compared with meta-analytic norms ${ }^{6}$ of perceived sleep continuity outcomes after CBT-I in people with insomnia, the change scores of the present study were comparable, but CBT-I participants had somewhat lower effect sizes ( sleep latency $=0.44 \mathrm{vs} 1.05$, wake after sleep onset $=0.83 \mathrm{vs}$ 1.03 , number of awakenings $=0.44$ vs 0.83 , subjective sleep quality $=1.02 \mathrm{vs} 1.44$ ) except for total sleep time, which was higher $(0.70$ vs 0.46$)$. There are several possible reasons for this. The sample of participants with COPD comprised $80 \%$ men, which may account for some of the difference in the effect sizes. Another is that the difference in outcome was related to features of COPD that were present throughout the intervention period. Evidence supporting this is that the effect sizes had greater accordance with previous studies of CBT-I in people with insomnia comorbid with other medical illnesses. ${ }^{13,17,21,22}$ CBT-I study participants had relatively low sleep latency (32 minutes) at baseline, which could have affected the sleep latency outcome. Finally, the sleep restriction provided in this study was probably not as aggressive as in some studies of CBT-I. Evidence supporting this is that the total sleep time after the intervention discussed was higher than would be expected after a maximal sleep restriction. 
With regard to the effect of CBT-I on fatigue, higher POMS-F scores were seen at baseline compared with levels reported in the literature about healthy older adults. ${ }^{44,54}$ Baseline CRQ-F scores were comparable with those previously found in elderly adults with COPD. ${ }^{55}$ Fatigue scores were reduced following the CBT-I intervention as indicated by the CRQ-F, although this was not evident in the POMS-F subscale. The observation of reduced fatigue is in accordance with Espie and colleagues, who reported significant reductions in fatigue after CBT-I in cancer patients. ${ }^{16}$ Clearly, issues of fatigue measurement are complex.

In the CBT-I group, no intervention effects on mood state or daytime functions were evident, in accordance with previous studies of older adults with insomnia in the context of other chronic illnesses..$^{15,21,22,53}$ An unexpected finding was that, compared with the CBT-I group, the WE group had improved anxiety and depression with much larger withingroup effect sizes. A possible reason is that the components of the WE addressed a number of major areas of interest to those with COPD, such as managing exacerbations and breathing techniques for minimizing breathlessness. WE could have increased perceived self-efficacy in achieving control over their COPD and consequently or separately improved mood. This observation is consistent with the findings of Kunik and colleagues, who reported improved anxiety and expression after eight sessions of WE in people with COPD. ${ }^{56}$

Limitations of this study include small sample size, lack of postintervention objective COPD disease severity measures, and lack of complete long-term follow-up. However, follow-up data on five participants in CBT-I were collected, 3 months or longer postintervention. It was found that the insomnia severity within group difference (mean [SD]) prepost CBT-I was virtually unchanged (0.000[3]), suggesting that results were lasting. Despite the limitations, this study provides valuable preliminary information about CBT-I for people with COPD and insomnia.

\section{Conclusion}

This study was not powered to test for statistically significant differences between the interventions, but the sleep and fatigue outcome data indicate a large within-group effect size, which could potentially translate into improved patient outcomes. These results must be interpreted with caution because of the small sample size. However, the data suggest that CBT-I is feasible as an option for insomnia in COPD and that it may have effects similar to those found in its use with older adults with insomnia comorbid with other illnesses.
A larger, adequately powered study will be necessary to address the efficacy of CBT-I for people with COPD.

\section{Acknowledgments}

This research was funded by NIH KO1 NR010749, NIH/ NNRR 5K30RR022271, and NIH/NINR 5 P30. The authors would like to thank the staff at the UIC Sleep Science Center for their assistance with this project.

\section{Disclosure}

The authors report no conflicts of interest in this work.

\section{References}

1. Bellia V, Catalano F, Scichilone N, et al. Sleep disorders in the elderly with and without chronic airflow obstruction: the SARA study. Sleep. 2003;26(3):318-323.

2. Klink M, Quan SF. Prevalence of reported sleep disturbances in a general adult population and their relationship to obstructive airways diseases. Chest. 1987;91(4):540-546.

3. Roth T. Hypnotic use for insomnia management in chronic obstructive pulmonary disease. Sleep Med. 2009;10(1):19-25.

4. Morin CM, Culbert JP, Schwartz SM. Nonpharmacological interventions for insomnia: a meta-analysis of treatment efficacy. Am J Psychiatry. 1994;151(8):1172-1180.

5. Morin CM, Colecchi C, Stone J, Sood R, Brink D. Behavioral and pharmacological therapies for late-life insomnia: a randomized controlled trial. JAMA. 1999;281(11):991-999.

6. Smith MT, Perlis ML, Park A, et al. Comparative meta-analysis of pharmacotherapy and behavior therapy for persistent insomnia. Am J Psychiatry. 2002;159(1):5-11.

7. Edinger JD, Wohlgemuth WK, Radtke RA, Marsh GR, Quillian RE. Cognitive behavioral therapy for treatment of chronic primary insomnia: a randomized controlled trial. JAMA. 2001;285(14):1856-1864.

8. Edinger JD, Olsen MK, Stechuchak KM, et al. Cognitive behavioral therapy for patients with primary insomnia or insomnia associated predominantly with mixed psychiatric disorders: a randomized clinical trial. Sleep. 2009;32(4):499-510.

9. Jacobs GD, Pace-Schott EF, Stickgold R, Otto MW. Cognitive behavior therapy and pharmacotherapy for insomnia: a randomized controlled trial and direct comparison. Arch Intern Med. 2004;164(17):1888-1896.

10. Sivertsen B, Omvik S, Pallesen S, et al. Cognitive behavioral therapy vs zopiclone for treatment of chronic primary insomnia in older adults: a randomized controlled trial. JAMA. 2006;295(24):2851-2858.

11. Siebern AT, Manber R. Insomnia and its effective non-pharmacologic treatment. Med Clin North Am. 2010;94(3):581-591.

12. Morin CM, Bootzin RR, Buysse DJ, et al. Psychological and behavioral treatment of insomnia: update of the recent evidence (1998-2004). Sleep. 2006;29(11):1398-1414.

13. Smith MT, Huang MI, Manber R. Cognitive behavior therapy for chronic insomnia occurring within the context of medical and psychiatric disorders. Clin Psychol Rev. 2005;25(5):559-592.

14. Perlis ML, Sharpe M, Smith MT, Greenblatt D, Giles D. Behavioral treatment of insomnia: treatment outcome and the relevance of medical and psychiatric morbidity. J Behav Med. 2001;24(3):281-296.

15. Lichstein KL, Wilson NM, Johnson CT. Psychological treatment of secondary insomnia. Psychol Aging. 2000;15(2):232-240.

16. Espie CA, Fleming L, Cassidy J, et al. Randomized controlled clinical effectiveness trial of cognitive behavior therapy compared with treatment as usual for persistent insomnia in patients with cancer. $J$ Clin Oncol. 2008;26(28):4651-4658.

17. Fiorentino L, McQuaid JR, Liu L, et al. Individual cognitive behavioral therapy for insomnia in breast cancer survivors: a randomized controlled crossover pilot study. Nat Sci Sleep. 2009;2010:1-8. 
18. Edinger JD, Wohlgemuth WK, Krystal AD, Rice JR. Behavioral insomnia therapy for fibromyalgia patients: a randomized clinical trial. Arch Intern Med. 2005;165(21):2527-2535.

19. Currie SR, Wilson KG, Pontefract AJ, deLaplante L. Cognitive-behavioral treatment of insomnia secondary to chronic pain. JConsult Clin Psychol. 2000;68(3):407-416

20. Jungquist CR, O'Brien C, Matteson-Rusby S, et al. The efficacy of cognitive-behavioral therapy for insomnia in patients with chronic pain Sleep Med. 2010;11(3):302-309.

21. Vitiello MV, Rybarczyk B, Von Korff M, Stepanski EJ. Cognitive behavioral therapy for insomnia improves sleep and decreases pain in older adults with co-morbid insomnia and osteoarthritis. J Clin Sleep Med. 2009;5(4):355-362.

22. Rybarczyk B, Stepanski E, Fogg L, et al. A placebo-controlled test of cognitive-behavioral therapy for comorbid insomnia in older adults. J Consult Clin Psychol. 2005;73(6):1164-1174.

23. Guilleminault C, Davis K, Huynh NT. Prospective randomized study of patients with insomnia and mild sleep disordered breathing. Sleep. 2008;31(11):1527-1533.

24. Manber R, Edinger JD, Gress JL, et al. Cognitive behavioral therapy for insomnia enhances depression outcome in patients with comorbid major depressive disorder and insomnia. Sleep. 2008;31(4):489-495.

25. Global Initiative for Chronic Obstructive Lung Disease (GOLD). Global Strategy for the Diagnosis, Management, and Prevention of Chronic Obstructive Pulmonary Disease. Bethesda, MD: GOLD; 2009.

26. Morin CM. Insomnia: Psychological Assessment and Management. New York, NY: Guilford Press; 1993.

27. Snaith RP. The Hospital Anxiety And Depression Scale. Health Qual Life Outcomes. 2003;1:29.

28. Miller MR, Hankinson J, Brusasco V, et al. Standardisation of spirometry. Eur Respir J. 2005;26(2):319-338.

29. Bastien $\mathrm{CH}$, Vallieres A, Morin CM. Validation of the Insomnia Severity Index as an outcome measure for insomnia research. Sleep Med. 2001;2(4):297-307.

30. Bjelland I, Dahl AA, Haug TT, Neckelmann D. The validity of the Hospital Anxiety and Depression Scale. An updated literature review. J Psychosom Res. 2002;52(2):69-77.

31. Rechtschaffen A, Kales A. A Manual of Standardized Terminology, Techniques, and Scoring Systems for Sleep Stages of Human Subjects. Bethesda, MD: US Department of Health, Education, and Welfare, Public Health Services - National Institutes of Health, National Institute of Neurological Diseases and Blindness, Neurological Information Network; 1968.

32. Buysse D, Reynolds CI, Monk T, Berman S, Kupfer D. The Pittsburgh Sleep Quality Index: A new instrument for psychiatric practice and research. Psychiatry Res. 1989;28:193-213.

33. Carpenter J,Andrykowski M. Psychometric evaluation of the Pittsburgh Sleep Quality Index. Journal of Psychomatic Research. 1998;45(1):5-12.

34. Coates TJ, Killen JD, George J, et al. Estimating sleep parameters: a multitrait-multimethod analysis. J Consult Clin Psychol. 1982;50(3): 345-352.

35. Guilleminault C, Clerk A, Black J, et al. Nondrug treatment trials in psychophysiologic insomnia. Arch Intern Med. 1995;155(8):838-844.

36. Sadeh A. The role and validity of actigraphy in sleep medicine: An update. Sleep Med Rev. 2011;15(4):259-267.

37. Morin CM, Stone J, Trinkle D, Mercer J, Remsberg S. Dysfunctional beliefs and attitudes about sleep among older adults with and without insomnia complaints. Psychol Aging. 1993;8(3):463-467.

International Journal of COPD

\section{Publish your work in this journal}

The International Journal of COPD is an international, peer-reviewed journal of therapeutics and pharmacology focusing on concise rapid reporting of clinical studies and reviews in COPD. Special focus is given to the pathophysiological processes underlying the disease, intervention programs, patient focused education, and self management protocols.
38. Morin CM, Blais F, Savard J. Are changes in beliefs and attitudes about sleep related to sleep improvements in the treatment of insomnia? Behav Res Ther. 2002;40(7):741-752.

39. Guyatt GH, Berman LB, Townsend M, Pugsley SO, Chambers LW. A measure of quality of life for clinical trials in chronic lung disease. Thorax. 1987;42:773-778.

40. Wijkstra PJ, TenVergert EM, Van Altena R, et al. Reliability and validity of the chronic respiratory questionnaire (CRQ). Thorax. 1994;49(5):465-467.

41. Meek PM, Nail LM, Barsevick A, et al. Psychometric testing of fatigue instruments for use with cancer patients. Nurs Res. 2000;49(4): 181-190.

42. Jaeschke R, Singer J, Guyatt GH. Measurement of health status. Ascertaining the minimal clinically important difference. Control Clin Trials. 1989;10(4):407-415

43. McNair D, Lorr M, Droppleman M. The Profile of Mood States. San Diego, CA: Educational and Industrial Testing Service; 1992.

44. Kaye JM, Lawton M, Gitlin L, et al. Older people's performance on the Profile of Mood States (POMS). Clin Gerontol. 1988;7:35-56.

45. Leidy NK. Psychometric properties of the Functional Performance Inventory in patients with chronic obstructive pulmonary disease. Nurs Res. 1999;48(1):20-28.

46. Larson JL, Kapella MC, Wirtz S, Covey MK, Berry J. Reliability and validity of the functional performance inventory in patients with moderate to severe chronic obstructive pulmonary disease. J Nurs Meas. 1998;6(1):55-73.

47. Perlis ML, Jungquist C, Smith MT, Posner D. Cognitive Behavioral Treatment of Insomnia. New York, NY: Springer; 2005.

48. Spielman AJ, Caruso LS, Glovinsky PB. A behavioral perspective on insomnia treatment. Psychiatr Clin North Am. 1987;10(4):541-553.

49. Edinger JD, Carney CE. Overcoming Insomnia: A Cognitive-Behavioral Therapy Approach Therapist Guide. 1st ed. New York, NY: Oxford University Press; 2008.

50. Di Marco F, Verga M, Reggente M, et al. Anxiety and depression in COPD patients: the roles of gender and disease severity. Respir Med. 2006;100(10):1767-1774

51. Fabbri L, Pauwels RA, Hurd SS. Global Strategy for the Diagnosis, Management, and Prevention of Chronic Obstructive Pulmonary Disease: GOLD Executive Summary updated 2003. COPD. 2004;1(1) 105-141; discussion 103-104.

52. Buysse D, Reynolds C III, Monk T, et al. Quantification of subjective sleep quality in healthy elderly men and women using the Pittsburgh Sleep Quality Index (PSQI). Sleep. 1991;14(4):331-338.

53. Rybarczyk B, Lopez M, Benson R, Alsten C, Stepanski E. Efficacy of two behavioral treatment programs for comorbid geriatric insomnia. Psychol Aging. 2002;17(2):288-298.

54. Nyenhuis D, Yamamoto C, Luchetta T, Terrien A, Parmentier A. Adult and geriatric normative data and validation of the profile of mood states. J Clin Psychol. 1999;55(1):79-86.

55. Sundararajan L, Balami J, Packham S. Effectiveness of outpatient pulmonary rehabilitation in elderly patients with chronic obstructive pulmonary disease. J Cardiopulm Rehabil Prev. 2010;30(2):121-125.

56. Kunik ME, Veazey C, Cully JA, et al. COPD education and cognitive behavioral therapy group treatment for clinically significant symptoms of depression and anxiety in COPD patients: a randomized controlled trial. Psychol Med. 2008;38(3):385-396.

This journal is indexed on PubMed Central, MedLine and CAS. The manuscript management system is completely online and includes a very quick and fair peer-review system, which is all easy to use. Visit http://www.dovepress.com/testimonials.php to read real quotes from published authors. 